\title{
Erratum to: Enhanced production of hyoscyamine and scopolamine from genetically transformed root culture of Hyoscyamus reticulatus $\mathbf{L}$. elicited by iron oxide nanoparticles
}

Fereshte Moharrami ${ }^{1} \cdot$ Bahman Hosseini $^{1} \cdot$ Ali Sharafi $^{2} \cdot$ Manouchehr Farjaminezhad $^{3}$

Published online: 19 April 2017

(C) The Society for In Vitro Biology 2017

Erratum to: In Vitro Cell Dev Biol -Plant

DOI 10.1007/s11627-017-9802-0

The correct names of the first and second authors are Fereshte Moharrami and Bahman Hosseini, respectively. The original article was corrected.

The original version of this article was revised: The correct names of the first and second authors are Fereshte Moharrami and Bahman Hosseini, respectively.

The online version of the original article can be found at http://dx.doi.org/ 10.1007/s11627-017-9802-0

Bahman Hosseini

b.hosseini@urmia.ac.ir

1 Horticultural Sciences Department, Faculty of Agriculture, Urmia University, Urmia, Iran

2 Pharmaceutical Biotechnology Research Center, School of Pharmacy, Zanjan University of Medical Sciences, Zanjan, Iran

3 Medicinal Plants Research Center, Ardabil Branch, Islamic Azad University, Ardabil, Iran 